\title{
The Galactic centre mini-spiral in the mm-regime
}

\author{
D. Kunneriath ${ }^{1,2,3}$, A. Eckart1,2, S. N. Vogel ${ }^{4}$, P. Teuben ${ }^{4}$, K. Mužicic ${ }^{5}$, R. Schödel ${ }^{6}$, M. García-Marín ${ }^{1}$, \\ J. Moultaka ${ }^{7}$, J. Staguhn ${ }^{4,8,9}$, C. Straubmeier ${ }^{1}$, J. A. Zensus ${ }^{2,1}$, M. Valencia-S. ${ }^{1,2}$, and V. Karas ${ }^{3}$ \\ 1 I. Physikalisches Institut, Universität zu Köln, Zülpicher Str. 77, 50937 Köln, Germany \\ e-mail: devaky@astro.cas.cz \\ 2 Max-Planck-Institut für Radioastronomie, Auf dem Hügel 69, 53121 Bonn, Germany \\ 3 Astronomical Institute, Academy of Sciences, Boční II 1401, 14100 Prague, Czech Republic \\ ${ }^{4}$ Department of Astronomy, University of Maryland, College Park, MD 20742-2421, USA \\ 5 Department of Astronomy and Astrophysics, University of Toronto, 50 St. George Str., Toronto ON M5S 3H4, Canada \\ ${ }^{6}$ Instituto de Astrofísica de Andalucía - CSIC, Glorieta de la Astronomía S/N, 18008, Spain \\ 7 LATT, Université de Toulouse, CNRS, 14 Avenue Edouard Belin, 31400 Toulouse, France \\ 8 NASA Goddard Space Flight Ctr., Greenbelt, MD 20771, USA \\ 9 Department of Physics and Astronomy, Johns Hopkins University, Baltimore, MD 21218, USA
}

Received 9 July 2011 / Accepted 2 December 2011

\begin{abstract}
Context. The mini-spiral is a feature of the interstellar medium in the central $\sim 2 \mathrm{pc}$ of the Galactic center. It is composed of several streamers of dust and ionised and atomic gas with temperatures between a few $100 \mathrm{~K}$ to $10^{4} \mathrm{~K}$. There is evidence that these streamers are related to the so-called circumnuclear disk of molecular gas and are ionized by photons from massive, hot stars in the central parsec.

Aims. We attempt to constrain the emission mechanisms and physical properties of the ionized gas and dust of the mini-spiral region with the help of our multiwavelength data sets.

Methods. Our observations were carried out at $1.3 \mathrm{~mm}$ and $3 \mathrm{~mm}$ with the $\mathrm{mm}$ interferometric array CARMA in California in March and April 2009, with the MIR instrument VISIR at ESO's VLT in June 2006, and the NIR Br $\gamma$ with VLT NACO in August 2009.

Results. We present high resolution maps of the mini-spiral, and obtain a spectral index of $0.5 \pm 0.25$ for Sgr A*, indicating an inverted synchrotron spectrum. We find electron densities within the range $0.8-1.5 \times 10^{4} \mathrm{~cm}^{-3}$ for the mini-spiral from the radio continuum maps, along with a dust mass contribution of $\sim 0.25 M_{\odot}$ from the MIR dust continuum, and extinctions ranging from $1.8-3$ at $2.16 \mu \mathrm{m}$ in the $\operatorname{Br} \gamma$ line.

Conclusions. We observe a mixture of negative and positive spectral indices in our $1.3 \mathrm{~mm}$ and $3 \mathrm{~mm}$ observations of the extended emission of the mini-spiral, which we interpret as evidence that there are a range of contributions to the thermal free-free emission by the ionized gas emission and by dust at $1.3 \mathrm{~mm}$.
\end{abstract}

Key words. accretion, accretion disks - black hole physics - Galaxy: nucleus - radio continuum: general - Galaxy: center

\section{Introduction}

The radio emission from the inner few parsecs of the Galactic centre (GC) region is dominated by the interstellar medium of the circumnuclear disk (CND) and the mini-spiral, the nuclear stellar cluster, and the supermassive black hole $\left(\sim 4 \times 10^{6} M_{\odot}\right)$ associated with the radio and infra-red source Sgr A* (Eckart \& Genzel 1996; Schödel et al. 2002; Ghez et al. 2009; Genzel et al. 2010, and references therein). The CND is assumed to be an association of clouds/filaments of dense $\left(10^{4}-10^{7} \mathrm{~cm}^{-3}\right)$ and warm (several $100 \mathrm{~K})$ molecular gas $\left(\sim 10^{5} M_{\odot}\right.$ of dust and gas, Christopher et al. 2005), which orbits the nucleus in a circular rotation pattern, with a sharp inner edge at $1.5 \mathrm{pc}$ that extends no further than $7 \mathrm{pc}$ from the centre (Guesten et al. 1987; Christopher et al. 2005; Montero-Castaño et al. 2009). The region inside this cavity contains atomic and ionized gas.

Several streamers of infalling gas and dust from the inner edge of the CND form the mini-spiral, which consists of four main components: the northern arm, the western arc, the eastern arm and the bar (labelled in Fig. 2, Ekers et al. 1983; Lo \& Claussen 1983; Zhao et al. 2009). These streams have been modelled as a bundle of three elliptical Keplerian orbits, with some significant deviations (Zhao et al. 2009, 2010), the northern and eastern arms being on highly elliptical orbits, while the western arc is nearly circular. The deviations from Keplerian motion have been assumed to be caused by stellar winds. The central region is also bright in the near-infrared (NIR) and midinfrared (MIR) regimes, where the stellar population dominates the NIR and warm dust emission accounting for MIR emission at $10 \mu \mathrm{m}$ (Becklin \& Neugebauer 1975; Becklin et al. 1978; Viehmann et al. 2006). Analyses of VLA observations at $5 \mathrm{GHz}$ (Brown et al. 1981) and Ne[II] line observations (Serabyn \& Lacy 1985; Serabyn et al. 1991) have also found peaks of emission coinciding with these $10 \mu \mathrm{m}$ thermal peaks.

The mini-spiral is a region with a complex temperature and density structure with temperatures ranging from a few hundred $\mathrm{K}$ in the dust component to $10^{4} \mathrm{~K}$ in the plasma component (Moultaka et al. 2005). Dust emission modelling of the central parsec region in the infrared $(4.8-20.0 \mu \mathrm{m})$ reveals temperatures of a few hundreds K with IRS $7(1300 \mathrm{~K})$ and IRS $3(600 \mathrm{~K})$ being the two hottest sources (Gezari et al. 1996; Moultaka et al. 2004, 2005). The source Sgr A* is 
Table 1. Log of the mm, NIR, and MIR observations.

\begin{tabular}{lcccc}
\hline \hline Telescope & Instrument/Array & $\lambda$ & $\begin{array}{c}\text { UT and JD } \\
\text { Start time }\end{array}$ & $\begin{array}{c}\text { UT and JD } \\
\text { Stop time }\end{array}$ \\
\hline CARMA & C array & \multirow{2}{*}{$3.0 \mathrm{~mm}$} & 2009 17 May 07:21:23.5 & 17 May 12:24:27.5 \\
& & & JD 2 454 968.806522 & JD 2 454 969.016985 \\
CARMA & D array & \multirow{2}{*}{$1.3 \mathrm{~mm}$} & 2009 28 Mar. 11:20:03.5 & 28 Mar. 14:39:18.5 \\
& & & JD 2 454 918.972263 & JD 2 454 919.110631 \\
CARMA & C array & \multirow{2}{*}{$1.3 \mathrm{~mm}$} & 2009 19 Apr. 10:07:21.5 & 19 Apr. 13:13:13.5 \\
& & & JD 2 454 940.921777 & JD 2 454 941.050851 \\
VLT & NACO & \multirow{2}{*}{$2.16 \mu \mathrm{m}$} & 2009 05 Aug. 22:59:14.59 & 05 Aug. 23:30:44.34 \\
& & & JD 2 455 049.457808 & JD 2 455 049.479680 \\
VLT & \multirow{2}{*}{$8.6 \mu \mathrm{m}$} & 2006 05 Jun. 04:53:43.77 & 05 Jun. 10:25:07.49 \\
& VISIR & & JD 2 453 891.703979 & JD 2 453 891.934114 \\
\hline
\end{tabular}

very bright at radio/mm wavelengths at a typical resolution of a few arcseconds. Interferometric observations at these wavelengths can separate the flux density contributions of Sgr A* and the mini-spiral region from the surrounding CND. Zhao et al. $(2009,2010)$ presented $1 \mathrm{~mm}$ maps of $\sim 0.15^{\prime \prime}$ resolution of the mini-spiral region, while Mauerhan et al. (2005) presented maps of the highest resolution yet achieved at $3 \mathrm{~mm}$ of $\sim 4$ " resolution.

The spectral index behaviour of the mini-spiral at these wavelengths has never been studied. We therefore embark on such an investigation in the present paper. Our work (the preliminary results of which were published in Kunneriath et al. 2011) complements the discussion of García-Marín et al. (2011), who presented sub-mm emission maps of the inner $37 \times 34 \mathrm{pc}^{2}$ of the GC region, including the CND and surrounding molecular clouds. These authors derived temperature maps of this region, which revealed dust temperatures between $10 \mathrm{~K}$ and $20 \mathrm{~K}$ in the CND and molecular clouds. Spectral index maps of the same region indicate that the spectral indices range from -0.8 to 1.0 in these low temperature regions, which has been interpreted as evidence of a combination of dust, synchrotron, and free-free emission. In the regions immediately surrounding $\mathrm{Sgr} \mathrm{A}^{*}$, they derived spectral indices of $-0.6<\alpha<0.0$, which was explained as a combination of 70-90\% synchrotron and 10-30\% dust and free-free emission. However, at sub-mm wavelengths, the emission of Sgr A* dominates the central few arcseconds, making an analysis of the emission mechanisms of the mini-spiral difficult at these wavelengths.

In the radio regime, Ekers et al. (1975) published a spectral index map of the Galactic centre region between $6 \mathrm{~cm}$ and $20 \mathrm{~cm}$ wavelengths, and obtained a non-thermal spectral index of -0.3 for the diffuse emission from Sgr A west, and a thermal spectrum for the Sgr A west spiral. However, at $20 \mathrm{~cm}$, the spiral features are not clearly resolved, and the emission could be optically thick at $\lambda \geq 6 \mathrm{~cm}$ (Mezger et al. 1989).

We present ( $\left.\sim 4^{\prime \prime}\right)$ high spatial resolution maps from observations of the Galactic centre region at 1.3 and $3 \mathrm{~mm}$ performed in 2009 using CARMA, yielding a spectral index map obtained at millimetre frequencies, a comparison to the MIR dust continuum and NIR Br $\gamma$ emission in the same region, and an analysis of the emission processes in the mini-spiral region. Section 2 is dedicated to a description of the observations and data reduction methods, while Sects. 3 and 4 provide the results and summary. Physical properties of the dust and gas in selected regions are calculated and tabulated in Table 2.

\section{Observations and data reduction}

\subsection{Millimetre observations}

The Combined Array for Research in mm-wave Astronomy $(\mathrm{CARMA})^{1}$ is a millimetre array consisting of 15 telescopes, including six $10.4 \mathrm{~m}$ telescopes with a half-power beamwidth $(H P B W)$ of $69^{\prime \prime}$ at $100 \mathrm{GHz}$ and $30^{\prime \prime}$ at $230 \mathrm{GHz}$, and nine $6.1 \mathrm{~m}$ telescopes with $H P B W$ of $115^{\prime \prime}$ at $100 \mathrm{GHz}$ and $50^{\prime \prime}$ at $230 \mathrm{GHz}$. Five array configurations are possible, ranging from A $(0.25-2 \mathrm{~km})$ to $\mathrm{E}(8-66 \mathrm{~m})$, and reaching an angular resolution of up to $0.15^{\prime \prime}$ at $230 \mathrm{GHz}$ in the A-array.

The observations at $3 \mathrm{~mm}(100 \mathrm{GHz})$ were made in May 2009 with CARMA in the C configuration with intermittent observations of 3C 279 for bandpass calibration and 1733-130 for phase and amplitude calibration. The only flagging done was to remove shadowing effects. At $1.3 \mathrm{~mm}(230 \mathrm{GHz})$, observations were made in March and April 2009 in the C and D configuration, using MWC349 for bandpass calibration and 1733-130 for phase and amplitude calibration. Owing to anomalous sytem temperature values, the complete antenna 7 dataset was flagged and antenna 14 was flagged from 11:50.9 to 12:10.0 and 10:00.0 to $11: 10.0 \mathrm{UT}$. The uv-coverages of the $\mathrm{C}$ array at $3 \mathrm{~mm}$ and the $\mathrm{D}$ array at $1.3 \mathrm{~mm}$ are shown in the Appendix.

Table 1 gives the details of observations with start and stop times. All observations were centred at $\alpha(\mathrm{J} 2000.0)=17: 45: 40.04$ and $\delta(\mathrm{J} 2000.0)=-29: 00: 28.09$. The data reduction was performed with Miriad, an interferometric data reduction package (Sault et al. 1995). The calibrated visibilities were inverted using the mosaic option and a CLEAN algorithm was applied to produce the final radio maps.

\subsection{MIR observations}

The observations at $8.6 \mu \mathrm{m}$ (PAH 1 filter with central wavelength $8.59 \mu \mathrm{m}, \Delta \lambda=0.42 \mu \mathrm{m})$ were carried out in June 2006 with the VLT Imager and Spectrometer for mid-Infrared (VISIR) at the European Southern Observatory (ESO) Very Large Telescope (VLT) Unit 3. The field of view of the combined mosaic image obtained by shifting and adding background subtracted images

1 Support for CARMA construction was derived from the states of California, Illinois, and Maryland, the Gordon and Betty Moore Foundation, the Kenneth T. and Eileen L. Norris Foundation, the Associates of the California Institute of Technology, and the National Science Foundation. Ongoing CARMA development and operations are supported by the National Science Foundation under a cooperative agreement, and by the CARMA partner universities. 
Table 2. Fluxes and calculated properties of selected mini-spiral regions.

\begin{tabular}{lccccccccc}
\hline \hline Region & $\begin{array}{c}S_{3 \mathrm{~mm}} \\
\mathrm{Jy}\end{array}$ & $\begin{array}{c}S_{1.3 \mathrm{~mm}} \\
\mathrm{Jy}\end{array}$ & $\begin{array}{c}S_{8.6 \mu \mathrm{m}} \\
\mathrm{Jy}\end{array}$ & $\begin{array}{c}S_{\mathrm{Br} \gamma} \\
\mathrm{Jy}\end{array}$ & $\begin{array}{c}E M \\
\times 10^{6} \mathrm{~cm}^{-6} \mathrm{pc}\end{array}$ & $\begin{array}{c}n_{\mathrm{e}} \\
\times 10^{4} \mathrm{~cm}^{-3}\end{array}$ & $\begin{array}{c}N_{\mathrm{Lyc}} \\
\times 10^{50} \mathrm{~s}^{-1}\end{array}$ & $\begin{array}{c}M_{\text {dust }} \\
M_{\odot}\end{array}$ & $\begin{array}{c}\alpha \\
3 \text { to } 1.3 \mathrm{~mm}\end{array}$ \\
\hline & \pm 0.03 & \pm 0.03 & \pm 5 & \pm 0.015 & \pm 0.8 & \pm 0.3 & \pm 0.6 & \pm 0.006 & \\
\hline 1 & 0.24 & 0.22 & 20.9 & 0.096 & 9.71 & 1.45 & 3.43 & 0.016 & $0.02_{-0.03}^{+0.08}$ \\
2 & 0.12 & 0.10 & 16.2 & 0.042 & 2.02 & 0.95 & 2.08 & 0.013 & $-0.09_{-0.05}^{+0.04}$ \\
3 & 0.15 & 0.15 & 23.6 & 0.080 & 6.07 & 1.15 & 2.57 & 0.018 & $-0.16_{-0.08}^{+0.03}$ \\
4 & 0.14 & 0.13 & 46.6 & 0.125 & 3.43 & 1.05 & 2.48 & 0.036 & $-0.07_{-0.09}^{+0.14}$ \\
5 & 0.08 & 0.07 & 21.6 & 0.062 & 1.99 & 0.79 & 1.48 & 0.017 & $-0.12_{-0.02}^{+0.05}$ \\
6 & 0.09 & 0.07 & 16.6 & 0.049 & 4.09 & 0.89 & 1.79 & 0.013 & $-0.10_{-0.06}^{+0.06}$ \\
\hline
\end{tabular}

Notes. Fluxes from different wavelengths, calculated emission measure, electron density, number of ionizing photons, dust mass, and spectral index (with its variation across the region) for selected regions from the mini-spiral. Positions of the selected regions are shown in Fig. 6. The second row gives the uncertainties in values.

obtained by dithering is $30^{\prime \prime} \times 30^{\prime \prime}$ at a pixel scale of $0.075^{\prime \prime}$ (Lagage et al. 2003). Further details of the observations, data reduction, and flux calibration are given in Schödel et al. (2007).

\subsection{NIR Bracketty}

The Brackett $\gamma$ images were obtained using the NB 2.166 filter of the NAOS/CONICA adaptive optics system at the ESO/VLT (Lenzen et al. 2003; Rousset et al. 2003), on August 5th, $2009^{2}$. Standard data reduction, including bad pixel correction, sky subtraction, flat field correction, and the detector-row cross-talk correction, was performed using IDL and DPUSER ${ }^{3}$.

The individual images were combined in a mosaic using a simple shift-and-add algorithm. Shifts between the individual exposures were determined by cross-correlation, performed by the jitter routine (part of the ESO eclipse package; Devillard 1997). The images were then shifted and median averaged using DPUSER. Point source subtraction was performed using StarFinder (Diolaiti et al. 2000). Since the FOV of the $\mathrm{Br} \gamma$ mosaic is significantly larger than the isoplanatic angle at this wavelength $\left(\sim 6^{\prime \prime}\right)$, we divided the mosaic into overlapping subimages. A local PSF extracted from each of the sub-images was used for PSF fitting and subtraction of stars (for a more detailed description of the procedure see Schödel 2010; Schödel et al. 2010).

Residuals at the positions of bright stars are mainly due to imperfections in PSF extraction, and extreme stellar crowding in the central parsec of the GC (Fig. 1). The photometric zeropoint was calculated using the precise $K_{\mathrm{S}}$-band photometry from Schödel et al. (2010) of 20 stars randomly distributed in the field. The $K$-band magnitudes are then converted to Janskys using the flux density of Vega in the $K_{\mathrm{S}}$-band (Allen 1976). An offset of 0.13 mag was applied to the $K_{\mathrm{S}}$-magnitudes to convert them to $\mathrm{Br} \gamma$ - magnitudes. This offset was calculated using the transmission curves of both the $K_{\mathrm{S}}$ and NB filters and assuming a given extinction law, total extinction, and blackbody temperature. The calculated magnitude offset was insensitive to the assumed extinction (which varied between $\mathrm{A}_{K_{\mathrm{S}}}=2.0-3.5$ ), blackbody temperature $\left(T=2 \times 10^{3}-3 \times 10^{4} \mathrm{~K}\right)$, and extinction law (exponent

\footnotetext{
${ }^{2}$ Based on observations made by ESO telescopes at Paranal Observatory, under the programme 083.B-0390(A).

${ }_{3}$ Developed by Ott; http://www . mpe.mpg.de/ ott/dpuser
}

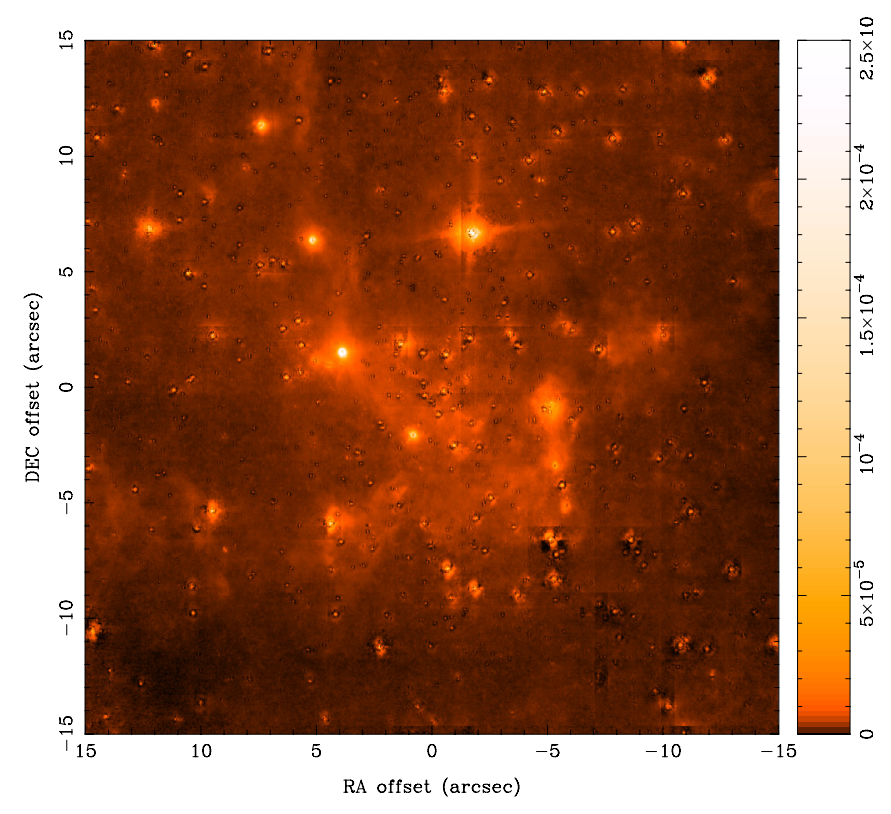

Fig. 1. Br $\gamma$ image of the central 30" mini-spiral region around Sgr A*. The colour bar indicates flux density units of Jy/pixel.

$\alpha=2.0-2.2$ ), and varied by $<0.015$ as these parameters were varied. At $\lambda \sim 2.30 \mu \mathrm{m}$, the SED of late-type stars deviates strongly from a blackbody because of the CO-bandhead absorption dip. However, this effect is limited to a small part of the $K_{\mathrm{S}}$ window and is not expected to be stronger than the one causing the blackbody temperature to vary by one order of magnitude. The measured $\mathrm{Br} \gamma$-band magnitudes were converted into Janskys, assuming 667 Jy for a source of magnitude zero (value for $K_{\mathrm{S}}$ filter from Cox 2000); we estimate that the uncertainty in this conversion factor is $<10 \%$.

\section{Results}

\subsection{Radio $3 \mathrm{~mm}$ and $1.3 \mathrm{~mm}$ maps}

We produced a $3 \mathrm{~mm}$ map of resolution $2.68^{\prime \prime} \times 1.71^{\prime \prime}$ $\left(\mathrm{PA}=-20.7^{\circ}\right)$ from the $\mathrm{C}$ array data of May 2009 (refer to Fig. 2). The primary beam $(H P B W)$ contains a total integrated flux of 13 Jy. Mezger et al. (1989) reported a total flux density 


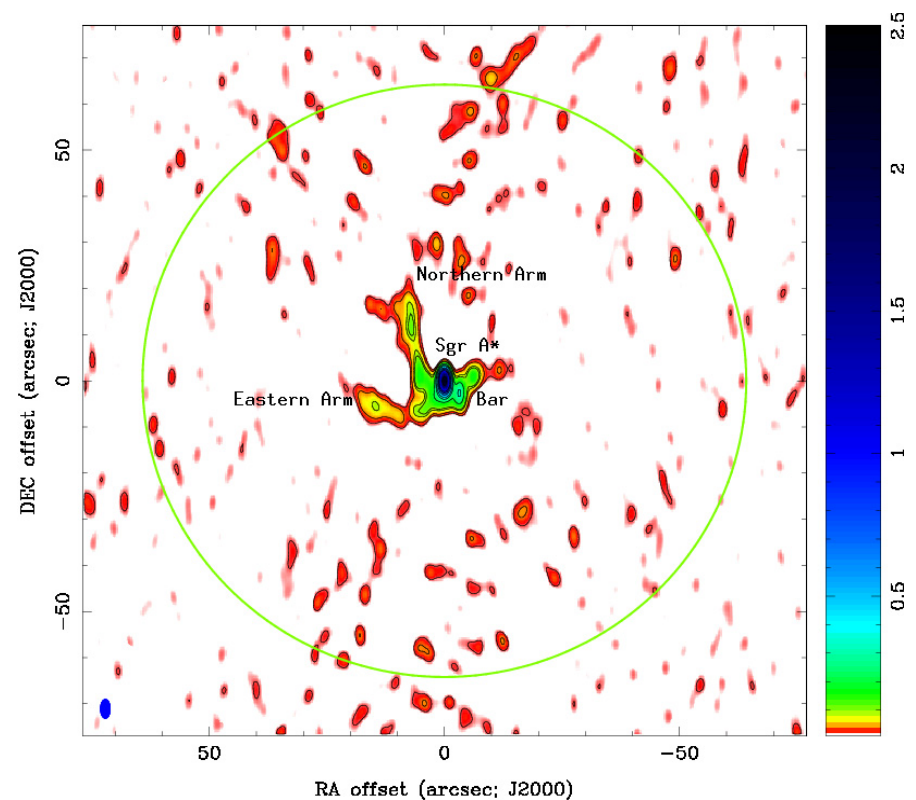

Fig. 2. Three-mm map of the mini-spiral region at resolution $2.68^{\prime \prime}$ by $1.71^{\prime \prime}\left(\mathrm{PA}=-20.7^{\circ}\right)$. The green circle marks the $H P B W$. Contour levels are $0.02,0.03,0.04,0.05,0.1,0.6,0.9,1.2,1.5$, and $2.5 \mathrm{Jy} /$ beam.

of $22 \mathrm{Jy}$ at $106 \mathrm{GHz}$ with the single dish IRAM $30 \mathrm{~m}$ telescope on Pico Veleta, Spain. The missing flux of $\sim 9 \mathrm{Jy}$ is due to the lower sensitivity of interferometric observations to the underlying extended thermal emission than for the brighter small-scale features of the mini-spiral. This is also seen in other interferometric observations, such as Shukla et al. (2004), who reported a total flux of $12 \mathrm{Jy}$ from the central $85^{\prime \prime}$ region using the OVRO array at $92 \mathrm{GHz}$ from a 6". $95 \times 33^{\prime \prime} 47\left(\mathrm{PA}=-5^{\circ}\right)$ map, and Wright et al. (1987) who reported a total flux of $12.6 \mathrm{Jy}$ at $86 \mathrm{GHZ}$ with the Hat Creek Interferometer. These agree with our flux measurements. We note that the missing flux does not pose a significant problem for our spectral index measurements, as explained in Sect. 3.3.

The uncertainty in the flux density measurements is given by $\sqrt{\left(\sigma_{\text {rms }}^{2}\right)+\left(\sigma_{\text {cal }} F_{v}\right)^{2}}$, where $\sigma_{\text {rms }}$ gives the rms noise of the map, $\sigma_{\text {cal }}$ gives the relative error in flux calibration, which we estimate to be $15 \%$ in our case, and $F_{v}$ is the flux density at frequency $v$.

The original $1.3 \mathrm{~mm}$ D array map of resolution $4.42^{\prime \prime} \times 1.23^{\prime \prime}$ $\left(\mathrm{PA}=1.0^{\circ}\right)$ is shown in Fig. 3 . The mini-spiral is clearly a complex region with several features of interest. To highlight these features, we present two maps in this paper. Figure 4 shows the $1.3 \mathrm{~mm} \mathrm{CD}$ array configuration map with a synthesized beam size corresponding to $1.2^{\prime \prime}$ emphasizing the compact structures in the region, which can be clearly distinguished from each other and from the extended emission of the mini-spiral. The other map (shown in Fig. 5), with a synthesized beam size corresponding to the lower angular resolution of $2.2^{\prime \prime}$ allows us to look at the more extended emission. The main features that we can see in the two maps are: the central non-thermal source Sgr A*, and most of the thermal mini-spiral, including the bar, eastern arm and parts of the northern arm. The western arc is resolved out in our maps.

To compare fluxes in different maps at different wavelengths, we chose six regions in the point-source-subtracted version of the extended emission map. Since the beam shape is elliptical, we chose elliptical regions (Fig. 6). The elliptical shape also allows us to fit the elongated structures in the mini-spiral region more closely, hence maximising the aperture area on source

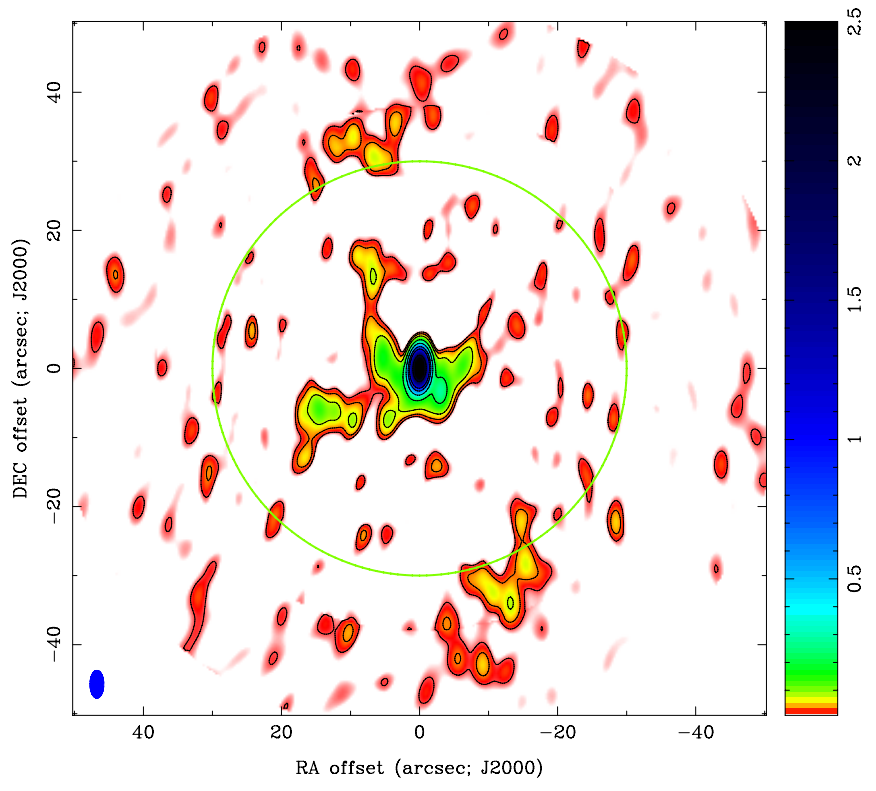

Fig. 3. A $1.3 \mathrm{~mm}$ map of the mini-spiral region at resolution $4.42^{\prime \prime}$ by $1.23^{\prime \prime}\left(\mathrm{PA}=1.0^{\circ}\right)$. The green circle marks the $H P B W$. Contour levels are $0.02,0.03,0.04,0.05,0.1,0.6,0.9,1.2,1.5$, and $2.5 \mathrm{Jy} / \mathrm{beam}$.

and avoiding any loss of flux from the source. The fluxes extracted from these sources in the $1.3 \mathrm{~mm}$ and $3 \mathrm{~mm}$ maps are tabulated in Table 2. Using the kinetic temperatures estimated by Zhao et al. (2010) in the mini-spiral arms from radio recombination lines ( $\mathrm{H} 92 \alpha$ and $\mathrm{H} 30 \alpha$ ), we calculated the properties of the selected regions including the emission measure and electron density from the free-free radio continuum emission flux densities at $3 \mathrm{~mm}$ (Panagia \& Walmsley 1978). We measured electron densities in the range $0.8-1.5 \times 10^{4} \mathrm{~cm}^{-3}$ in these regions, with the highest electron densities and emission occurring in the IRS 13 region. Zhao et al. (2010) obtained slightly higher values of number densities of $3-21 \times 10^{4} \mathrm{~cm}^{-3}$ for the same regions. However, our values are comparable to values of number densities of the order of $2 \times 10^{4} \mathrm{~cm}^{-3}$ obtained from radio continuum measurements in Brown et al. (1981) and Lo \& Claussen (1983) and from $\mathrm{Pa} \alpha$ measurements in Scoville et al. (2003). We can conclude that the derived values are consistent with previously published results within the errors.

\subsection{Spectral index}

Our spectral index map (to be published in the upcoming conf. proc. Kunneriath et al. 2012) covers the inner $40^{\prime \prime} \times 40^{\prime \prime}$ of the GC region, which includes the northern arm, eastern arm, the bar and the central bright radio source Sgr A* and was produced from the 3 and $1.3 \mathrm{~mm}$ images.

We used the notation $\mathbf{S}_{v} \sim v^{\alpha}$, where $\alpha$ is the spectral index and $\mathrm{S}_{v}$ is the flux density at frequency $v$. We convolved our $3 \mathrm{~mm}$ and $1.3 \mathrm{~mm}$ maps to an angular resolution of $4^{\prime \prime} \times 2^{\prime \prime}$, after which a primary beam correction was applied. We applied a flux density cutoff of $0.01 \mathrm{Jy}$ to both the images to exclude regions of low flux density to create masked images at both frequencies. By multiplying these masked images, we created a final mask, which was then used to obtain the spectral index map of the region. The masking process ensures that the spectral index is well-defined at all points in the masked region by avoiding any division by low flux values. With the calculated flux density calibration accuracy $\sim 15 \%$, we measured the 

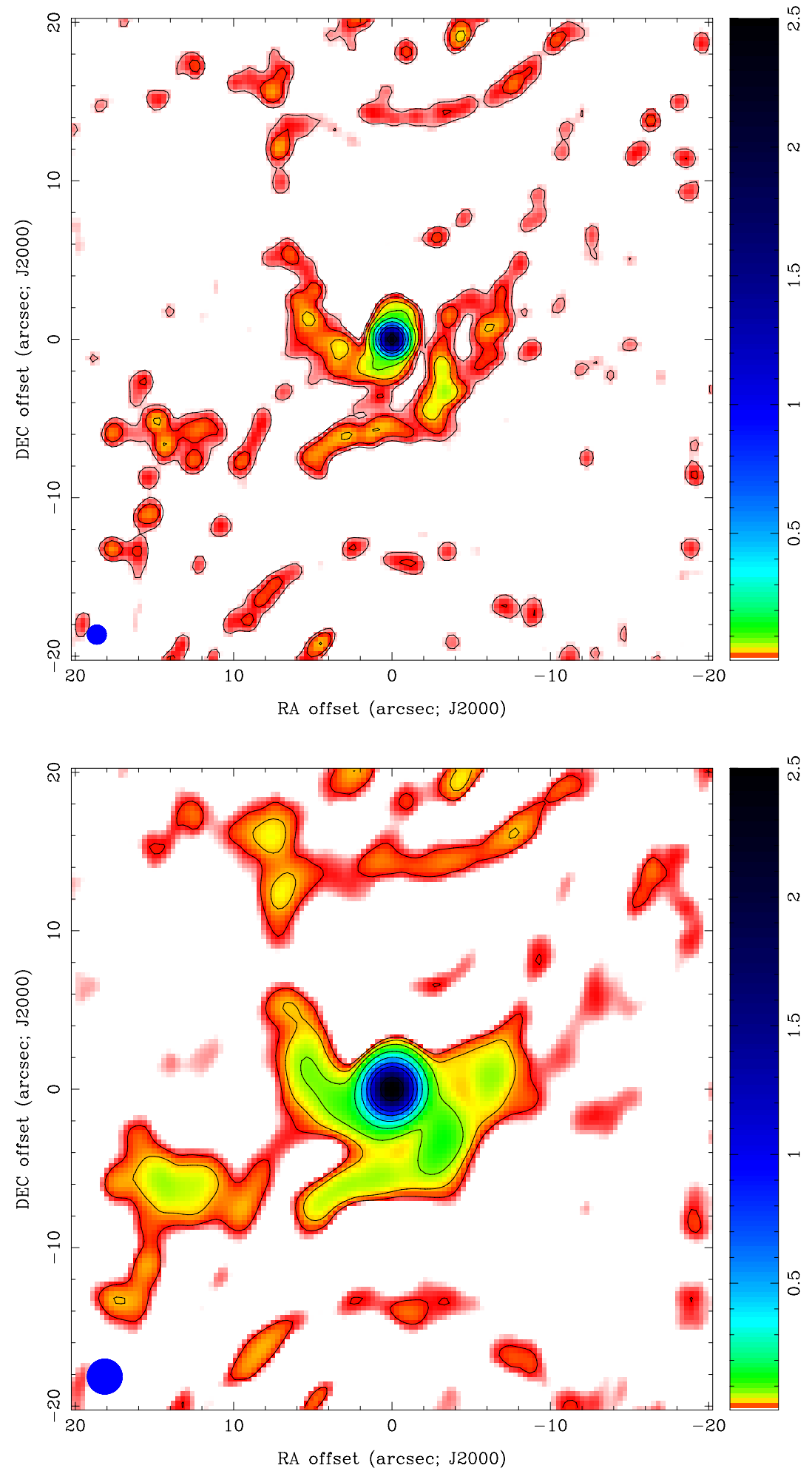

Fig. 4. The mini-spiral region image obtained from $1.3 \mathrm{~mm} \mathrm{CD}$ array configuration with a synthesised circular beam size of $1.2^{\prime \prime}$. Contour levels are $0.015,0.02$, $0.03,0.04,0.05,0.1,0.6,0.9,1.2,1.5$, and $2.5 \mathrm{Jy} /$ beam.

Fig. 5. The same as in Fig. 4 but with a synthesised circular beam size of $2.2^{\prime \prime}$. The different resolutions are to highlight emission from the compact and extended features in the region, as explained in Sect. 3.1.

uncertainty in the spectral index values to be $\delta \alpha \sim 0.25$ from $\delta \alpha=1 / \ln (v 1 / v 2) \times \sqrt{(\delta S 1 / S 1)^{2}+(\delta S 2 / S 2)^{2}}$, derived from the spectral index equation.

We obtained a spectral index of $\sim 0.5 \pm 0.25$ for Sgr A*, indicating an inverted synchrotron spectrum, which agrees with the results of Falcke et al. (1998) and Yusef-Zadeh et al. (2006). The mini-spiral region around $\mathrm{Sgr} \mathrm{A}^{*}$ shows a mixture of positive and negative spectral indices. Free-free thermal bremsstrahlung emission is indicated by the $\sim-0.1$ spectral index value, around the bar and parts of the northern arm. The steeper spectral indices of $\sim-0.5$ could be a result of unresolved flux in the $1.3 \mathrm{~mm}$ map, in particular in the northern arm of the mini-spiral. The positive indices in the spectral index map in the mini-spiral region, around the edges of the bar and most of the eastern arm, on the other hand, could be indicators of dust emission that starts to become significant at wavelengths $\leq 1.3 \mathrm{~mm}$. The regions of excess emission approximately coincide with locations of marginally cooler (at least by $10 \mathrm{~K}$ ) dust, as seen in the colour temperature map derived from $12.5 \mu \mathrm{m}$ and $20.3 \mu \mathrm{m}$ maps by Cotera et al. (1999), who found that the overall temperature in the mini-spiral 


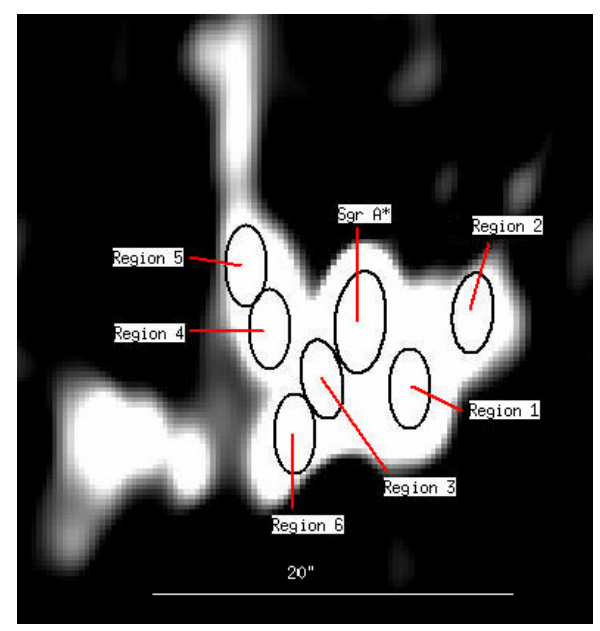

Fig. 6. The elliptical regions used to extract fluxes from the central 20" region of the GC from the multi-wavelength datasets, marked on the $1.3 \mathrm{~mm}$ radio continuum map of resolution $4^{\prime \prime} \times 2^{\prime \prime}\left(\mathrm{PA}=0^{\circ}\right)$.

is of the order of $\sim 200 \mathrm{~K}$. This indicates that we may have an unspecified amount of dust (of unknown mass and filling factor) at temperatures between $30 \mathrm{~K}$ and $200 \mathrm{~K}$, with larger-sized dust grains. Moultaka et al. (2009), who found evidence of intrinsic $\mathrm{CO}$ absorption at $4.6 \mu \mathrm{m}$ in the mini-spiral region, suggest that the presence of lower temperature material in the region is not inconsistent with the higher overall temperature, since compact dusty structures with high optical depths can have travel times of the order of $10^{3}$ years, which is comparable to photoevaporation timescales of $10^{3}-10^{5}$ years, allowing the cooler material to travel through the central parsec.

\subsection{Uncertainty in spectral index}

Spectral index maps are very sensitive to zero-spacing flux density offsets, in particular for radio interferometric observations, and if the observed wavelengths are as close as in our case. However, by choosing the D configuration at $1.3 \mathrm{~mm}$ we cover the range in angular resolution obtained in the $\mathrm{C}$ configuration at $3 \mathrm{~mm}$ very well. In the $\mathrm{C}$ configuration at $100 \mathrm{GHz}$, the angular resolution ranges from $2.16^{\prime \prime}$ for the shortest baseline of $30 \mathrm{~m}$ to $25.16^{\prime \prime}$ for the longest baseline of $350 \mathrm{~m}$, while in the $\mathrm{D}$ configuration at $230 \mathrm{GHz}$, the angular resolution ranges from $2.18^{\prime \prime}$ for the shortest baseline of $11 \mathrm{~m}$ to $29.74^{\prime \prime}$ for the longest baseline of $150 \mathrm{~m}$. Thus the effects of missing flux at short spacings can be assumed to be similar at both frequencies. The uvcoverages at both frequencies, given in the Appendix, demonstrate this similarity.

The spectral index map derived from this data contains valuable information about the flux density differences between both observing frequencies. This can be discussed in terms of emission mechanisms and resolution effects. The similarities between the maps indicate that we detect mainly emission from the compact components from within the mini-spiral, which has been successfully detected by all $\mathrm{mm}$-interferometers (see references in Sect. 3.1). We found that the median spectral index we obtained for these compact features is -0.1 , in full agreement with free-free emission. If the sources suffered an increasing amount of resolution on the CARMA baselines we would expect much steeper spectral index values. This is not observed, with the exception of the northern arm (see Sect. 3.2).

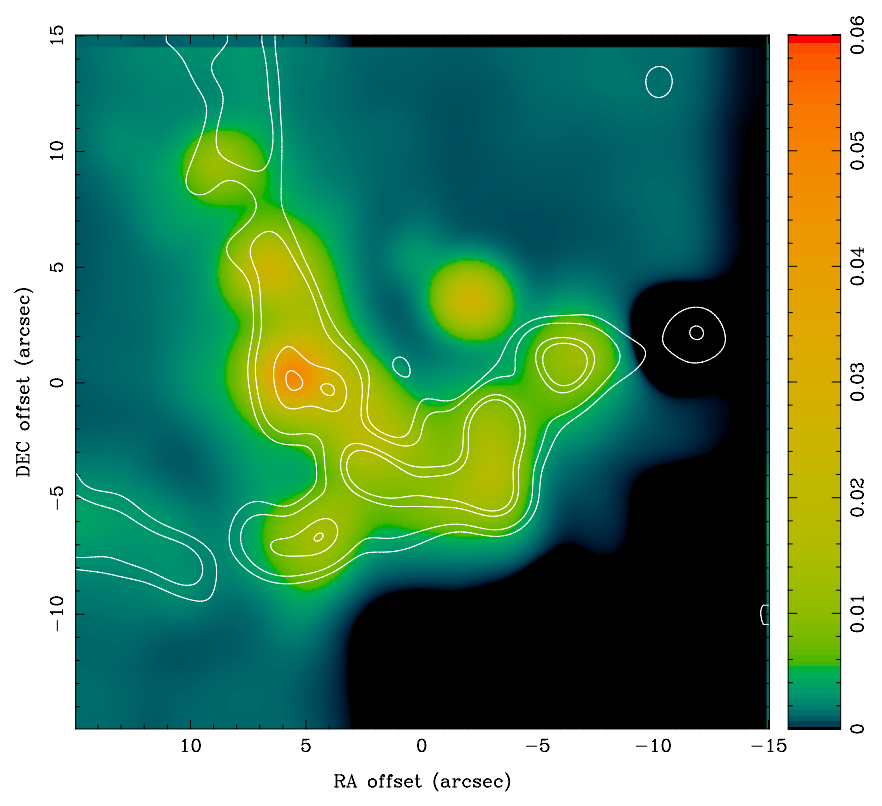

Fig. 7. MIR $8.6 \mu \mathrm{m}$ map overlaid with a $3 \mathrm{~mm}$ point source subtracted map of synthesised circular beam size of $2^{\prime \prime}$. Contour levels are at 0.02 , $0.035,0.08,0.1$, and $0.2 \mathrm{Jy} /$ beam.

\subsection{MIR map}

The MIR map at $8.6 \mu$ m reveals extended dust emission in the central parsec region. A strong correlation was observed between the warm dust emission at MIR $(12.4 \mu \mathrm{m})$ wavelengths and the ionized gas emission at radio wavelengths (VLA $2 \mathrm{~cm}$ ) by Gezari \& Yusef-Zadeh (1991), indicating that the dust and gas are mixed in the region, with the point sources IRS 1, 2, and 9 coincident in both maps and significant displacements in the position of IRS 13 and 21 between the two which could be due to dust displacement produced by stellar winds. An overlay of our high resolution $\mathrm{C}$ array $3 \mathrm{~mm}$ map over the $8.6 \mu \mathrm{m}$ map (at $2^{\prime \prime}$ resolution) is shown in Fig. 7, which confirms this correlation, with the radio contours tracing out the dust in the MIR quite well.

The uncertainties in flux density measurements are obtained from the rms error and flux calibration error (estimated to be $<10 \%$ ). Fluxes from the same regions selected in the radio continuum maps are extracted. The dust masses for the regions are calculated using

$M_{\mathrm{d}}=\frac{F(v) D^{2}}{B\left(v, T_{\mathrm{d}}\right)} \frac{4 a}{3 Q(v)} \rho$,

where $F(v)$ is the flux density, $D$ is the distance to the region, $a$, $Q(v)$, and $\rho$ are the dust parameters grain size, emissivity, and grain density, respectively, and $B(v, T)$ the Planck function at dust temperature $T_{\mathrm{d}}$, which we assume to be $200 \mathrm{~K}$ (Cotera et al. 1999). Dust grain parameters were obtained from Rieke et al. (1978). The masses are tabulated in Table 2.

\subsubsection{Dust-to-gas ratio}

The $3 \mathrm{~mm}$ extended emission map and the $8.6 \mu \mathrm{m}$ map were converted to the same pixel scale and shifted to match each other (using the position of Sgr $\mathrm{A}^{*}$ in the MIR from Schödel et al. 2007), to within an accuracy of $0.075^{\prime \prime}$ and used to obtain the dust-to-gas brightness ratio map shown in Fig. 8. The dark regions in the map indicate higher dust-to-gas ratios, while the 


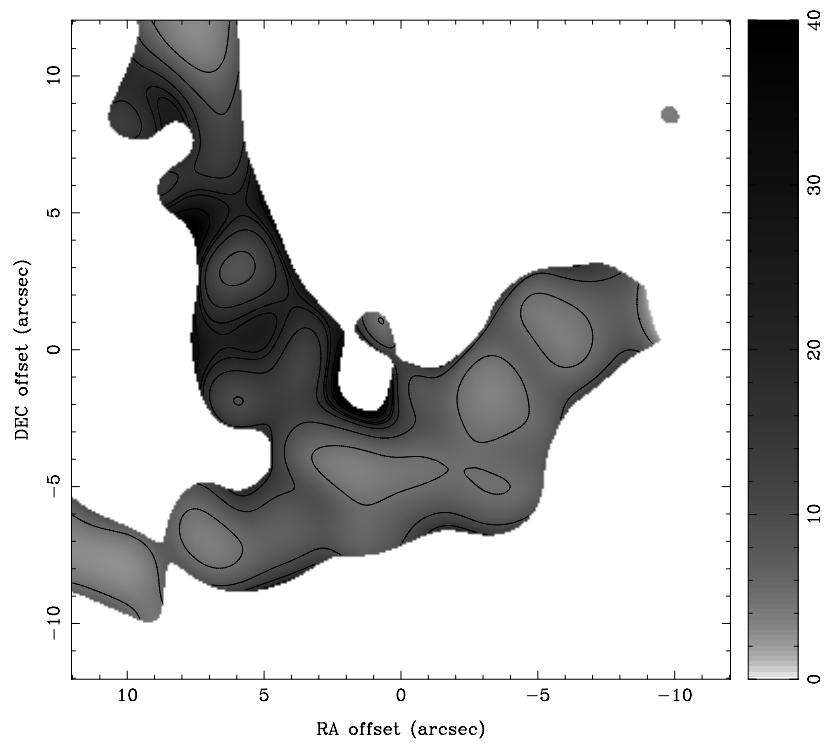

Fig. 8. Dust to gas ratio map with a synthesised circular beam size of $2^{\prime \prime}$ - High at IRS 10W, and IRS 1W (Northern Arm), low at IRS 13 (Bar).

lighter regions corresponds to less dust, with the values ranging from $\sim 10$ in the IRS 13 region to $\sim 30$ in the IRS $1 \mathrm{~W}$ region. The higher ratios in the northern arm than in the bar are consistent with the explanation that the ionized bar of the mini-spiral region is dust depleted by an outflow/wind from the central ionizing source Sgr A* (Gezari \& Yusef-Zadeh 1991).

\subsection{Bracketty map}

The Br $\gamma$ hydrogen recombination line at $2.16 \mu \mathrm{m}$ is a useful indicator of the ionized gas in the mini-spiral region, which traces out the same ionized emission seen in the radio continuum maps at $3 \mathrm{~mm}$. Neugebauer et al. (1978) report that most of the background flux at $2.2 \mu \mathrm{m}$ is from the stars in the region and estimate the free-free emission contribution to the continuum radiation at $2.2 \mu \mathrm{m}$ to be negligible. Therefore, after removing the strong stellar sources using a point-source subtraction method described in Sect. 2.3, we derived a reliable estimate of the emission line fluxes in the region. Similarities between the observed structures of the emission at $\mathrm{Br} \gamma$ and $3 \mathrm{~mm}$ wavelengths suggest that they largely trace emission from the same compact sources in the region. This allows us to use the CARMA $3 \mathrm{~mm}$ map to derive $5 \mathrm{GHz}$ continuum and $\mathrm{Br} \gamma$ line flux maps, respectively, as described below.

Assuming an optically thin free-free emission spectral index of -0.1 between $5 \mathrm{GHz}$ and $100 \mathrm{GHz}$, we scaled our $100 \mathrm{GHz}$ CARMA map to obtain a $5 \mathrm{GHz}$ flux density map. The expected $\mathrm{Br} \gamma$ line intensity can then be calculated from the $5 \mathrm{GHz}$ radio continuum flux density using the formula (Glass 1999)

$I(\mathrm{Br} \alpha)=2.71 \times 10^{-14}\left(\frac{T}{10^{4} \mathrm{~K}}\right)^{-0.85}\left(\frac{v}{\mathrm{GHz}}\right)^{0.1} F_{\nu}(\mathrm{Jy}) \mathrm{Wm}^{-2}$.

From the ratio of observed to expected flux density, we obtained an estimate of the extinction in the mini-spiral region at $2.16 \mu \mathrm{m}$. The extinction ranges from 1.8-3.0 mag in the mini-spiral arms, with a mean extinction of $2.30 \pm 0.16 \mathrm{mag}$. This is in good agreement with Schödel (2010), who reported a median extinction value of $\mathrm{A}_{K \mathrm{~s}}=2.74 \pm 0.30 \mathrm{mag}$, using $H-K$ colours. It is also in good agreement with Brown et al. (1981) and Scoville et al. (2003), who derived extinction values from the radio continuum emission and the $\mathrm{Br} \gamma$ and $\mathrm{Pa} \alpha$ line emission, respectively.

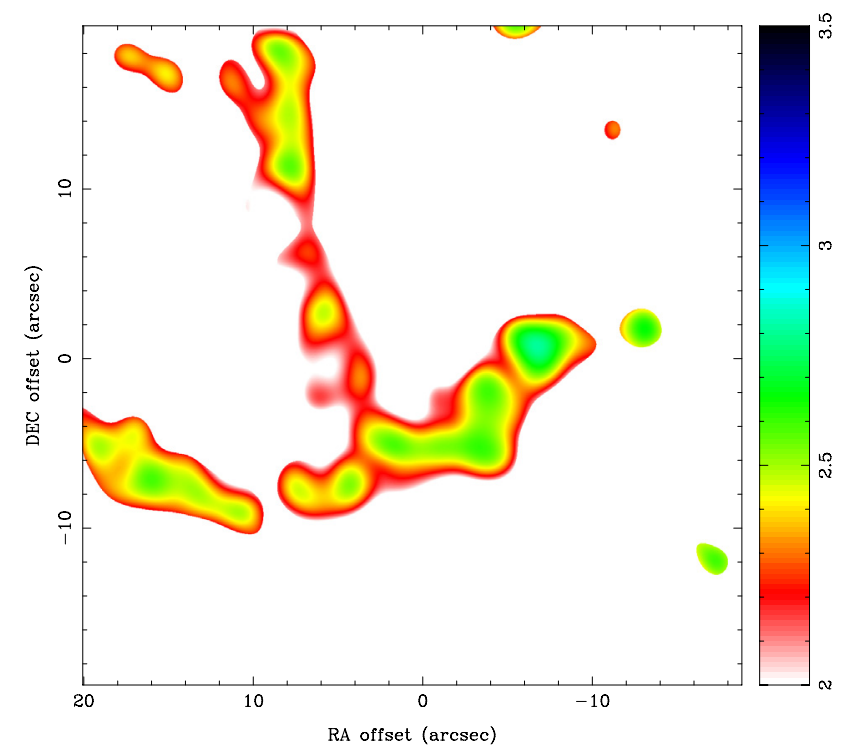

Fig. 9. Extinction map at $2.16 \mu \mathrm{m}$ (synthesized circular beam size of $2^{\prime \prime}$ ).

Figure 9 gives the extinction map at $2.16 \mu \mathrm{m}$. Following Ho et al. (1990), the number of ionizing photons was inferred from the expected Br $\gamma$ flux densities, which are tabulated in Table 2.

\section{Summary}

We have studied the mini-spiral at the Galactic centre at multiple wavelengths, including radio continuum maps at 3 and $1.3 \mathrm{~mm}$, MIR continuum at $8.6 \mu \mathrm{m}$, and the $\mathrm{Br} \gamma$ line emission at $2.16 \mu \mathrm{m}$. We have presented high resolution maps of both this mini-spiral and $\mathrm{Sgr} \mathrm{A}^{*}$ at $100 \mathrm{GHz}$ and $230 \mathrm{GHz}$. The spectral index map indicates that a mixture of various emission mechanisms operate in the central few parsecs of the GC, with an inverted synchrotron spectrum of $\sim 0.5$ of Sgr A*, thermal free-free emission spectral indices of -0.1 , and a possible contribution of dust emission as indicated by the positive thermal indices $\sim 1.0$. We attributed the positive spectral indices to a possible dust contribution that is evident at wavelengths $\leq 1.3 \mathrm{~mm}$, probably from large dust grains (Draine \& Lee 1984).

For selected regions in the mini-spiral arms, we extracted flux densities from the different wavelengths and inferred the following:

- The physical properties of the ionized gas such as emission measure are $2-10 \times 10^{6} \mathrm{~cm}^{-6} \mathrm{pc}$ and the electron densities are $0.8-1.5 \times 10^{4} \mathrm{~cm}^{-3}$ according to the $3 \mathrm{~mm}$ radio continuum map.

- The dust masses inferred from the $8.6 \mu \mathrm{m}$ MIR map, with a total dust mass contribution of $\sim 0.25 M_{\odot}$ from the minispiral arms, and a dust-to-gas brightness ratio map indicating dust depletion along the bar, with higher ratios along the northern arm.

- We inferred extinctions of $1.8-3.0$ at $2.16 \mu \mathrm{m}$ from a comparison of observed $\mathrm{Br} \gamma$ flux densities to the expected flux densities inferred from the free-free radio emission and the Lyman continuum emission rate derived from the expected $\operatorname{Br} \gamma$ flux density.

Acknowledgements. D. Kunneriath and M. Valencia-S. are members of the International Max Planck Research School (IMPRS) for Astronomy and Astrophysics at the MPIfR and the Universities of Bonn and Cologne. R.S. 
acknowledges support by the Ramón y Cajal programme by the Ministerio de Ciencia y Innovación of the government of Spain. Macarena Garcia-Marin is supported by the German federal department for education and research (BMBF) under the project numbers: 50OS0502 and 50OS0801. Part of this work was supported by the COST Action MP0905: Black Holes in a violent Universe and PECS project No. 98040. This research has made use of NASA's Astrophysics Data System.

\section{Appendix}

Figures A.1 and A.2 show the uv-coverage of the C array at $3 \mathrm{~mm}$ and the D array at $1.3 \mathrm{~mm}$. To derive reliable maps and determine the spectral index from the interferometric data, the uv-coverage of the maps must match each other very closely at different wavelengths. Since they correspond to different configurations of the CARMA array at different wavelengths, they cannot be exactly identical. However, they are comparable in uvdistance and appear to be similar enough to produce maps of comparable fluxes, using the data reduction method described in Sect. 2.1.

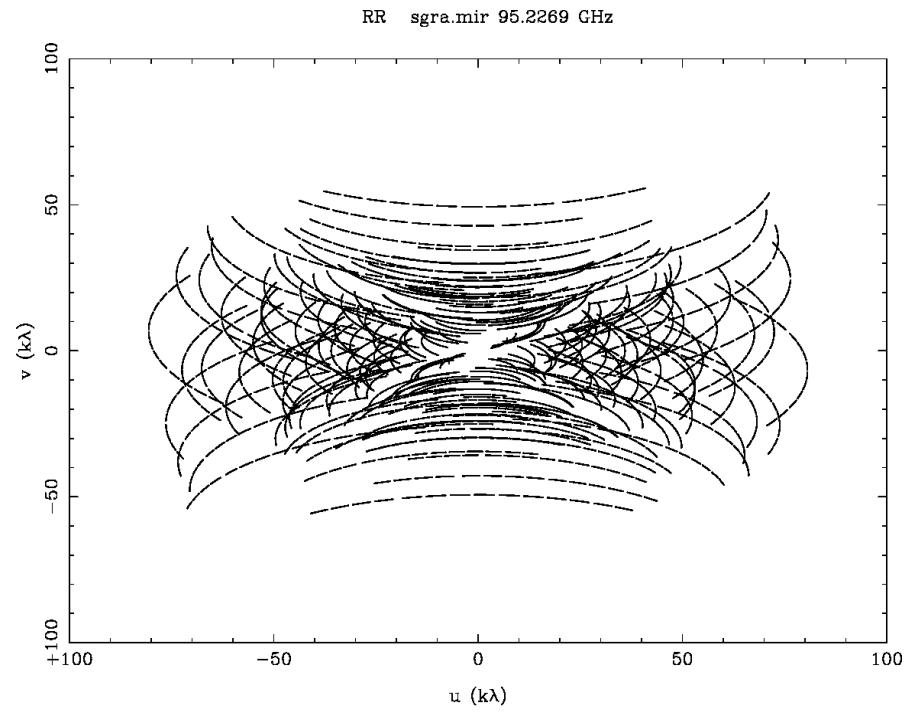

Fig. A.1. $\mathrm{C}$ array UV coverage at $3 \mathrm{~mm}$.

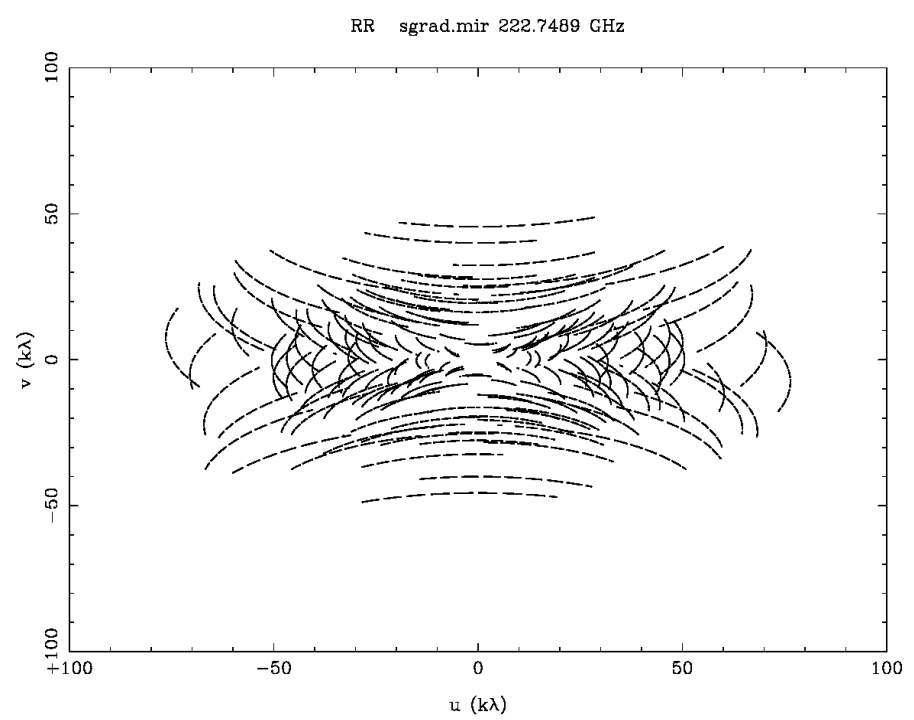

Fig. A.2. D array UV coverage at $1.3 \mathrm{~mm}$.

\section{References}

Allen, C. W. 1976, Astrophysical Quantities (London: Athlone), 3rd edn. Becklin, E. E., \& Neugebauer, G. 1975, ApJ, 200, L71

Becklin, E. E., Matthews, K., Neugebauer, G., \& Willner, S. P. 1978, ApJ, 219, 121

Brown, R. L., Johnston, K. J., \& Lo, K. Y. 1981, ApJ, 250, 155

Christopher, M. H., Scoville, N. Z., Stolovy, S. R., \& Yun, M. S. 2005, ApJ, 622, 346

Cotera, A., Morris, M., Ghez, A. M., et al. 1999, in The Central Parsecs of the Galaxy, ed. H. Falcke, A. Cotera, W. J. Duschl, F. Melia, \& M. J. Rieke, ASP Conf. Ser., 186, 240

Devillard, N. 1997, The Messenger, 87, 19

Diolaiti, E., Bendinelli, O., Bonaccini, D., et al. 2000, in SPIE Conf. Ser. 4007, ed. P. L. Wizinowich, 879

Draine, B. T., \& Lee, H. M. 1984, ApJ, 285, 89

Eckart, A., \& Genzel, R. 1996, Nature, 383, 415

Ekers, R. D., Goss, W. M., Schwarz, U. J., Downes, D., \& Rogstad, D. H. 1975, A\&A, 43, 159

Ekers, R. D., van Gorkom, J. H., Schwarz, U. J., \& Goss, W. M. 1983, A\&A, 122,143

Falcke, H., Goss, W. M., Matsuo, H., et al. 1998, ApJ, 499, 731

García-Marín, M., Eckart, A., Weiss, A., et al. 2011, in ASP Conf. Ser. 439, ed. M. R. Morris, Q. D. Wang, \& F. Yuan, 315

Genzel, R., Eisenhauer, F., \& Gillessen, S. 2010, Rev. Mod. Phys., 82, 3121

Gezari, D., \& Yusef-Zadeh, F. 1991, in ASP Conf. Ser. 14, ed. R. Elston, 214

Gezari, D., Dwek, E., \& Varosi, F. 1996, in Unsolved Problems of the Milky Way, ed. L. Blitz, \& P. J. Teuben, IAU Symp., 169, 231

Ghez, A., Morris, M., Lu, J., et al. 2009, Astronomy, 2010, 89

Glass, I. S. 1999, Handbook of Infrared Astronomy (Cambridge University Press)

Guesten, R., Genzel, R., Wright, M. C. H., et al. 1987, ApJ, 318, 124

Ho, P. T. P., Beck, S. C., \& Turner, J. L. 1990, ApJ, 349, 57

Kunneriath, D., Eckart, A., Zamaninasab, M., et al. 2011, in ASP Conf. Ser. 439 , ed. M. R. Morris, Q. D. Wang, \& F. Yuan, 327

Kunneriath, D., Eckart, A., Vogel, S. N., et al. 2012, in AHAR 2011, J. Phys. Conf. Ser.

Lagage, P., Pel, J., Claret, A., et al. 2003, in SPIE Conf. Ser. 4841, ed. M. Iye, \& A. F. M. Moorwood, 923

Lenzen, R., Hartung, M., Brandner, W., et al. 2003, in SPIE Conf. Ser. 4841, ed. M. Iye, \& A. F. M. Moorwood, 944

Lo, K. Y., \& Claussen, M. J. 1983, Nature, 306, 647

Mauerhan, J. C., Morris, M., Walter, F., \& Baganoff, F. K. 2005, ApJ, 623, L25

Mezger, P. G., Zylka, R., Salter, C. J., et al. 1989, A\&A, 209, 337

Montero-Castaño, M., Herrnstein, R. M., \& Ho, P. T. P. 2009, ApJ, 695, 1477

Moultaka, J., Eckart, A., Viehmann, T., et al. 2004, A\&A, 425, 529

Moultaka, J., Eckart, A., Schödel, R., Viehmann, T., \& Najarro, F. 2005, A\&A, 443,163

Moultaka, J., Eckart, A., \& Schödel, R. 2009, ApJ, 703, 1635

Neugebauer, G., Becklin, E. E., Matthews, K., \& Wynn-Williams, C. G. 1978, ApJ, 220, 149

Panagia, N., \& Walmsley, C. M. 1978, A\&A, 70, 411

Rieke, G. H., Telesco, C. M., \& Harper, D. A. 1978, ApJ, 220, 556

Rousset, G., Lacombe, F., Puget, P., et al. 2003, in SPIE Conf. Ser. 4839, ed. P. L. Wizinowich, \& D. Bonaccini, 140

Sault, R. J., Teuben, P. J., \& Wright, M. C. H. 1995, in Astronomical Data Analysis Software and Systems IV, ed. R. A. Shaw, H. E. Payne, \& J. J. E. Hayes, ASP Conf. Ser., 77, 433

Schödel, R. 2010, A\&A, 509, A58

Schödel, R., Ott, T., Genzel, R., et al. 2002, Nature, 419, 694

Schödel, R., Eckart, A., Mužić, K., et al. 2007, A\&A, 462, L1

Schödel, R., Najarro, F., Muzic, K., \& Eckart, A. 2010, A\&A, 511, A18

Scoville, N. Z., Stolovy, S. R., Rieke, M., Christopher, M., \& Yusef-Zadeh, F. 2003, ApJ, 594, 294

Serabyn, E., \& Lacy, J. H. 1985, ApJ, 293, 445

Serabyn, E., Lacy, J. H., \& Achtermann, J. M. 1991, ApJ, 378, 557

Shukla, H., Yun, M. S., \& Scoville, N. Z. 2004, ApJ, 616, 231

Viehmann, T., Eckart, A., Schödel, R., Pott, J., \& Moultaka, J. 2006, ApJ, 642, 861

Wright, M. C. H., Genzel, R., Güsten, R., \& Jaffe, D. T. 1987, in The Galactic Center, ed. D. C. Backer, AIP Conf. Ser., 155, 133

Yusef-Zadeh, F., Roberts, D., Wardle, M., Heinke, C. O., \& Bower, G. C. 2006, ApJ, 650, 189

Zhao, J.-H., Morris, M. R., Goss, W. M., \& An, T. 2009, ApJ, 699, 186

Zhao, J.-H., Blundell, R., Moran, J. M., et al. 2010, ApJ, 723, 1097 